\title{
Unmodulated spin chains as universal quantum wires
}

\author{
Antoni Wójcik, ${ }^{1}$ Tomasz Luczak,, , 冈 Paweł Kurzyński, ${ }^{1}$ Andrzej Grudka, ${ }^{1}$ Tomasz Gdala, ${ }^{2}$ and Małgorzata Bednarska ${ }^{2}$ \\ ${ }^{1}$ Faculty of Physics, Adam Mickiewicz University, Umultowska 85, 61-614 Poznań, Poland. \\ ${ }^{2}$ Faculty of Mathematics and Computer Science, Adam Mickiewicz University, Umultowska 87, 61-614 Poznań, Poland.
}

(Dated: May 11, 2005)

\begin{abstract}
We study a quantum state transfer between two qubits interacting with the ends of a quantum wire consisting of linearly arranged spins coupled by an excitation conserving, time-independent Hamiltonian. We show that if we control the coupling between the source and the destination qubits and the ends of the wire, the evolution of the system can lead to an almost perfect transfer even in the case in which all nearest-neighbour couplings between the internal spins of the wire are equal.
\end{abstract}

PACS numbers: 03.67.Hk, 03.67.Pp, 05.50.+q

The problem of designing quantum networks which enable efficient high-fidelity transfer of quantum states has recently been addressed by a number of authors (see [123]). Ideally, such a network should meet both the simplicity and the minimal control requirements. By simplicity we mean that the network consists of typical elements coupled in a standard way so that a few networks can be combined together to form more complex systems. The minimal control requirement says that the transmission of a quantum state through the network should be possible without performing many control operations (as switching interactions on and off, measuring, encoding and decoding, etc.). A 1D quantum network (quantum wire) which fulfills both above requirements was proposed by Bose [1] who considered a spin chain with the nearest neighbour Heisenberg Hamiltonian; here the transmission of quantum state between the ends of the chain was achieved simply by a free evolution of the network. However, as was shown by Bose, if all neighbour couplings have the same strength the fidelity of a transmission decreases with the chain length $n$. A similar model (with the Heisenberg Hamiltonian replaced by $\mathrm{XY}$ one) was considered by Christandl et al. in [2]. They show that one can transfer quantum states through arbitrary long chains if spin couplings are carefully chosen in a way depending on the chain length $n$ (see also [3-8]). Note however that this approach does not meet the simplicity requirement since one cannot merge several "modulated" quantum wires into a longer one.

Here, we study a transfer of quantum states between two qubits attached to the ends of a quantum wire consisting of $n$ linearly arranged spins. In order to fulfill the requirement of simplicity we assume that all couplings between neighbouring spins forming the quantum wire are the same (and equal to 1), while the couplings between the source and the destination qubits and the ends of the wire are equal to $a$. We show that one can significantly improve of the fidelity of the transfer be-

${ }^{*}$ Corresponding author. Phone: +48 (61) 829-5394, fax: +48 (61) 829-5315. E-mail: tomasz@amu.edu.pl tween the source and the destination qubits by selecting the value of $a$ appropriately. In particular, choosing $a$ small enough, one can achieve a transfer whose fidelity can be arbitrarily close to one, even for large $n$.

We assume that the Hamiltonian of the whole system of $n+2$ qubits conserves the number of excitation (e.g., it is a XY Hamiltonian), so the state $\bigotimes_{j=0}^{n+1}|0\rangle_{j}$ is its eigenstate. Let $F_{a b}$ denote the fidelity of the transfer of of an arbitrary state $|\phi\rangle=a|0\rangle+b|1\rangle$ from the source $(j=0)$ to destination $(j=n+1)$ qubit and let $F=F_{01}$ stand for the fidelity of the state $|1\rangle$ transfer. It is easy to check that the average fidelity $\left\langle F_{a b}\right\rangle$, where the average is taken over all possible values of $a$ and $b$, is uniquely determined by $F$, namely

$$
\left\langle F_{a b}\right\rangle=\frac{1}{3}+\frac{1}{6}(1+F)^{2} .
$$

Thus, from now on, we shall consider only the evolution of the system with the initial state given by $|\Psi(0)\rangle=|1\rangle_{0}|0\rangle_{1} \cdots|0\rangle_{n+1}$, i.e., the wire is in the polarized state. Then, the state space is spanned by vectors $|p\rangle=\bigotimes_{j=0}^{n+1}\left|\delta_{j p}\right\rangle_{j}, p=0, \ldots, n+1$, and the Hamiltonian $H(a)$ of the system can be written as

$$
H(a)=a(|0\rangle\langle 1|+| n\rangle\langle n+1|)+\sum_{p=1}^{n-1}|p\rangle\langle p+1|+\text { h.c. },
$$

where, let us recall, both the source and the destination qubits are coupled to the quantum wire with strength $a$, while all couplings among spins of the quantum wire are taken to be one. If $a^{2} \neq 2$, then all the eigenvalues of $H(a)$ are of the form $\lambda=2 \cos \gamma$, where $\gamma$ is a solution of either of the two following equations $(\mu= \pm 1)$ :

$$
\mu \cot (\gamma) \cot ^{\mu}\left(\frac{n+1}{2} \gamma\right)=\frac{a^{2}}{2-a^{2}}
$$

The eigenvector $\left|v^{(\gamma)}\right\rangle$ corresponding to the eigenvalue $2 \cos \gamma$ has coordinates $v_{k}^{(\gamma)}=\left\langle k \mid v^{(\gamma)}\right\rangle$ given by

$$
v_{0}^{(\gamma)}=\frac{a}{c} \sin \gamma
$$




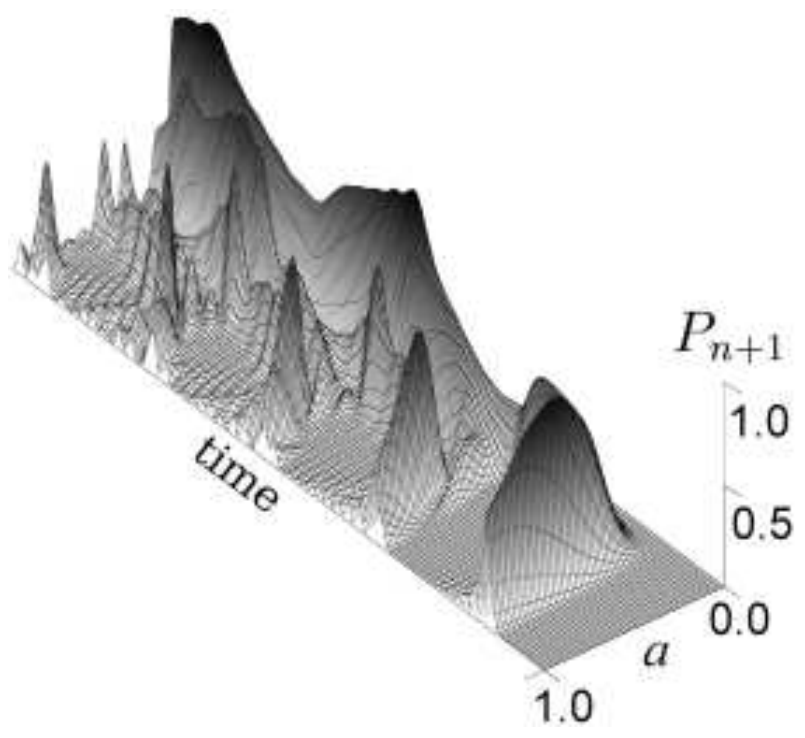

FIG. 1: The evolution of $P_{n+1}(t)$ for the system of 30 spins for $t \in[0,150]$.

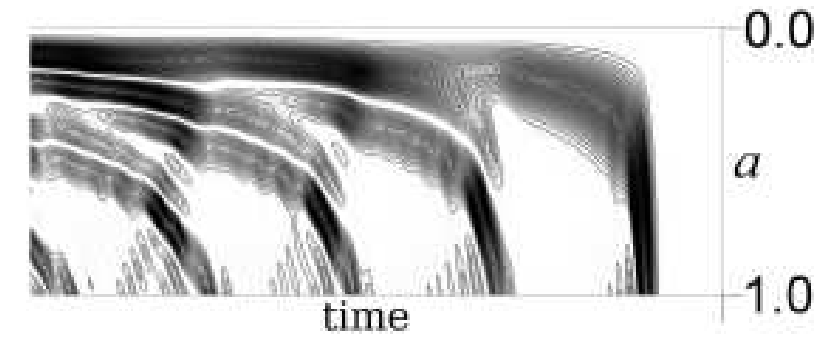

FIG. 2: The density plot for the graph in Figure 1.

$$
v_{k}^{(\gamma)}=\frac{1}{c}\left(\sin ((k+1) \gamma)+\left(1-a^{2}\right) \sin ((k-1) \gamma)\right),
$$

for $k=1, \ldots, n$, and

$$
v_{n+1}^{(\gamma)}=\mu v_{0}^{(\gamma)},
$$

where $c=c(a)$ is defined as

$$
c^{2}=(n+1)\left(2\left(1-a^{2}\right) \cos ^{2} \gamma+a^{4} / 2\right)+2 a^{2}-a^{4} .
$$

Let $P_{j}(t)=|\langle j \mid \Psi(t)\rangle|^{2}$ denote the probability of the excitation of the $j$ th spin, if the initial state of the system is $|1\rangle_{0}|0\rangle_{1} \cdots|0\rangle_{n+1}$ (i.e., $P_{0}(0)=1$ ). Figure 1 shows the dependence of $P_{n+1}(t)$ on $a$ for the first period of the evolution of a system of 30 spins, Figure 2 presents the density version of the same graph, while Figure 3 shows the projection of the evolution along the time axis. As clearly seen in Figure 3, decreasing $a$ from 1 to about 0.6 significantly improves the fidelity of transfer; if we decrease $a$ even further, then the fidelity drops down but for small $a$ it grows again approaching 1 as $a \rightarrow 0$.

In order to better understand Figure 3 we graph the effect of decreasing $a$ on the eigenvalue spectrum of the whole system as well as on the eigenvectors population

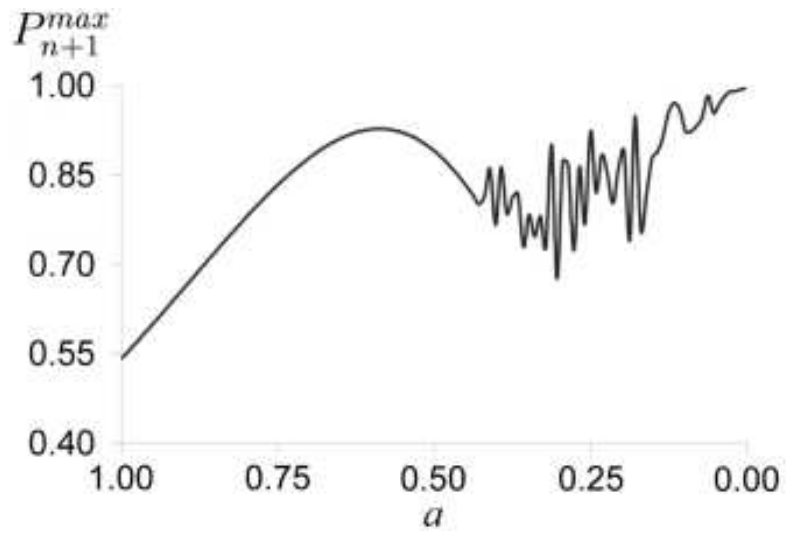

FIG. 3: The dependence of $P_{n+1}^{\max }=\max \left\{P_{n+1}(t): t \in\right.$ $[0,20000]\}$ on $a$.

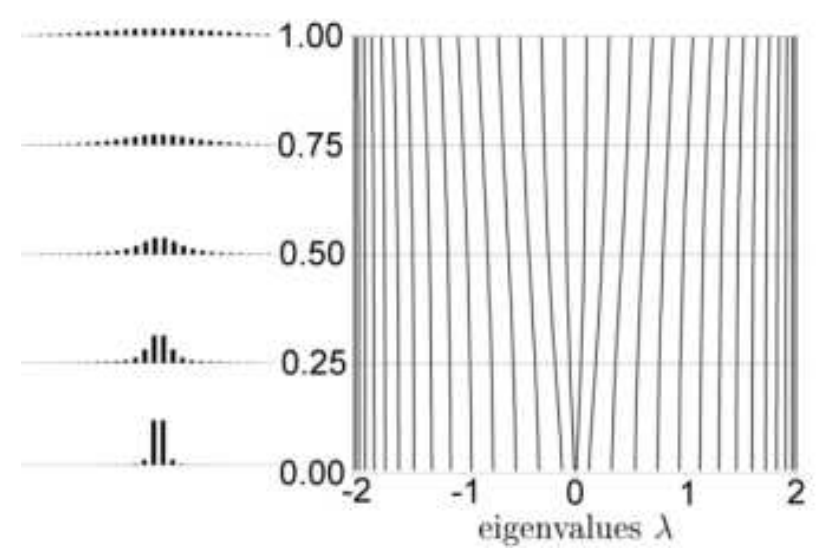

FIG. 4: The dependence of the eigenvalues of $H(a)$ on $a$ (the right hand side of the figure) and the eigenvector populations $\left\{|\langle v(\lambda) \mid \Psi(0)\rangle|^{2}\right\}_{\lambda}$ (on the left).

$\left|v_{0}^{(\gamma)}\right|^{2}$ (Figure 4). For $a \sim 1$ the spectrum is nearly harmonic in the vicinity of 0 but the distribution of the eigenvectors is broad and the contribution from the anharmonic part of the spectrum spoils the transfer. If $a$ decreases the eigenvectors distribution narrows so the anharmonic contribution drops down; consequently the transfer fidelity increases. On the other hand, for very small $a$, the harmonicity of the spectrum in the vicinity of zero brakes down, which reduces the fidelity of the transfer.

For small values of $a$ the behaviour of the system depends strongly on the parity of $n$. If $a \rightarrow 0+$, then the initial state $|\Psi(0)\rangle$ is concentrated basically on two eigenvectors for $n$ even (see Figure 44); if $n$ is odd, then the evolution of the system takes place in a three-dimensional space. More specifically, let $\hat{\lambda}_{a}$ be the smallest positive eigenvalue of $H(a)$. If $n$ is even and $a \rightarrow 0+$, then for the two eigenvectors $|x\rangle=\left|v\left(\hat{\lambda}_{a}\right)\right\rangle,|y\rangle=\left|v\left(-\hat{\lambda}_{a}\right)\right\rangle$ corresponding to the eigenvalues $\pm \hat{\lambda}_{a}$, we have $x_{0}=y_{0}=$ $x_{n+1}=-y_{n+1} \sim 1 / \sqrt{2}$. Consequently, an almost perfect transfer of states between qubits 0 and $n+1$ oc- 


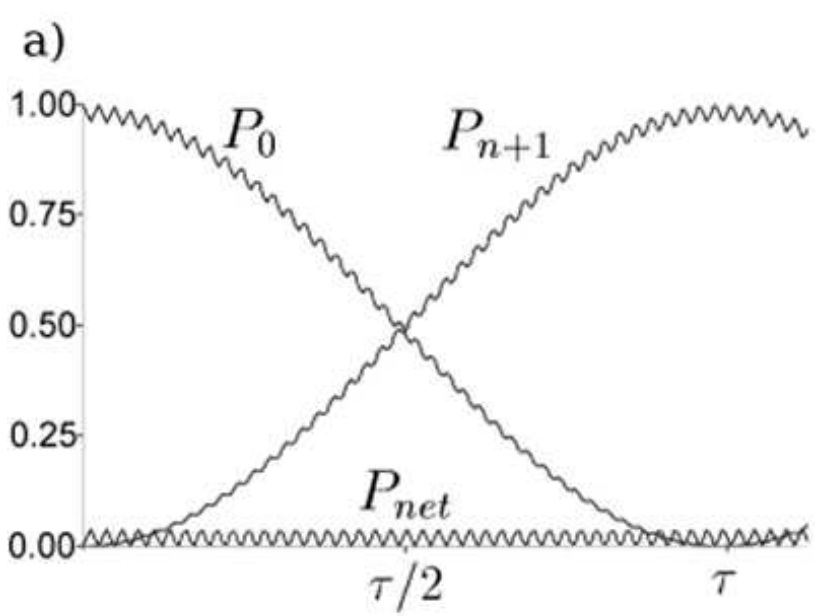

b)

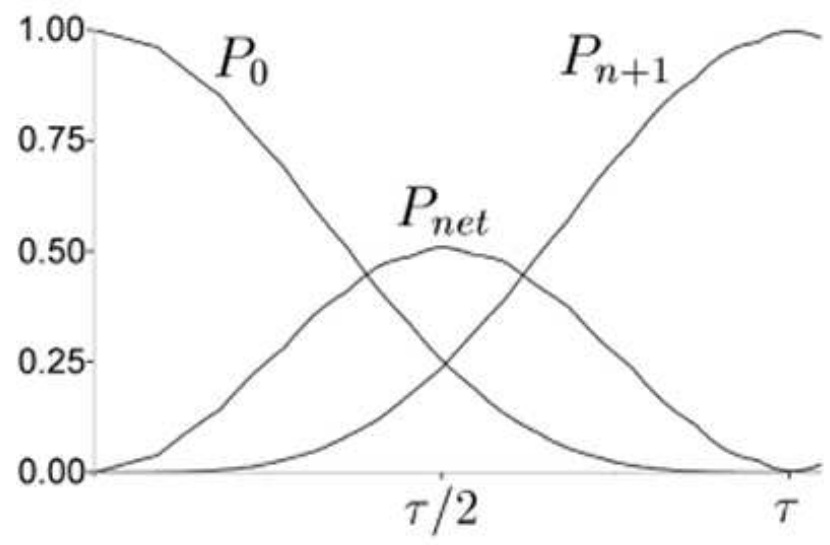

FIG. 5: The evolution of $P_{0}(t), P_{n+1}(t), P_{n e t}(t)$, for $a=0.01$ and (a) $n=198$; (b) $n=199$. If $n=198$, then $\lambda_{a} \sim 10^{-4}$ and $\tau \sim 16000$; for $n=201$ we have $\lambda_{a}=1.41 \cdot 10^{-3}$ and $\tau \sim 2200$.

curs after time $\tau \sim \pi /\left(2 \hat{\lambda}_{a}\right)$. For odd $n$ 's, the spectrum of $|\Psi(0)\rangle$ is concentrated on three eigenvectors: besides $|x\rangle=\left|v\left(\hat{\lambda}_{a}\right)\right\rangle$ and $|y\rangle=\left|v\left(-\hat{\lambda}_{a}\right)\right\rangle$, we need to take into account the eigenvector $|z\rangle=|v(0)\rangle$ corresponding to the eigenvalue zero. Then, $z_{0}=\left|z_{n+1}\right| \sim 1 / \sqrt{2}$, $x_{0}=y_{0}=\left|x_{n+1}\right|=\left|y_{n+1}\right| \sim 1 / 2$, and the sign of $z_{n+1}$ is opposite to the signs of both $x_{n+1}$ and $y_{n+1}$. In this case an almost perfect transfer from the source to the destination qubits occurs after time $\tau \sim \pi / \hat{\lambda}_{a}$. Let us mention that a similar phenomenon of transferring the states between two qubits weakly interacting with a network has been recently observed by Li et al. [9] and Plenio and Semiao [10], who consider transferring qubit states through a ladder and a cycle, respectively.

Straightforward computations show that the fidelity of the transfer scales as $1-O\left(a^{2} n\right)$ and it grows to one only when $a \ll 1 / \sqrt{n}$. Moreover, we have $\hat{\lambda}_{a} \sim a^{2}$ for an even $n$, and $\hat{\lambda}_{a} \sim 2 a / \sqrt{n}$ when $n$ is odd. Hence, the time of the transfer is $\Theta\left(a^{-2}\right)$ for even $n$, and $\Theta(\sqrt{n} / a)$ for odd $n$. Thus, for $a=\delta / \sqrt{n}$, an almost perfect $\left(F \approx 1-\delta^{2}\right)$ transfer is possible for $\delta \ll 1$ in a time which scales linearly with the quantum wire length $n$. The speed of the transfer $n / \tau$ is approximated by $2 \delta / \pi$ and $\delta^{2} / \pi$ when $n$ is odd and even, respectively. Thus, for small $a$, a quantum wire with odd $n$ ensures more effective transfer. In Figure 5 the evolution of the excitation probabilities of the source qubit $P_{0}(t)$, the destination qubit $P_{n+1}(t)$ and the total probability of the excitation of the wire qubits $P_{n e t}(t)=\sum_{j=1}^{n} P_{j}(t)$, are presented for $a=0.01$ and $n=198,199$. Note that if $n$ is even, the spins of the wire remain almost unexcited during the evolution, i.e., the source and destination qubits evolve like two isolated and directly coupled qubits. Thus, the evolution of a system reminds Rabi oscillations between the source and destination qubits, despite the fact that they lie in a significant distance from each other.

Finally, we remark that if $n$ is even and $a \rightarrow 0+$, after time $t \sim \tau / 2$ the state of the system is close to $\frac{1}{\sqrt{2}}\left(|0\rangle_{0}|1\rangle_{n+1}+|1\rangle_{0}|0\rangle_{n+1}\right)|0\rangle_{1} \cdots|0\rangle_{n}$. Thus, such an unmodulated quantum wire can also be used to generate entanglement between the source and destination qubits.

In conclusion, we have shown that one can significantly improve a transfer of the qubit states between the ends of a quantum wire by controlling the coupling on its ends. In particular, an unmodulated spin chain of arbitrary length $n$ can be used as a universal quantum wire to transfer of quantum states with arbitrary high fidelity in a time which scales linearly with $n$.

The authors wish to thank the State Committee for Scientific Research (KBN) for its support: A.W. and A.G. were supported by grant 0 T00A 003 23; T.E., T.G., and M.B. by grant 1 P03A 02527.
[1] S. Bose, Phys. Rev. Lett. 91, 207901 (2003).

[2] M. Christandl, N. Datta, A. Ekert, A.J. Landahl, Phys. Rev. Lett. 92, 187902 (2004).

[3] C. Albanese, M. Christandl, N. Datta, A. Ekert, Phys. Rev. Lett. 93, 230502 (2004).

[4] M-H. Yung, S. Bose, Phys. Rev. A 71, 032310 (2005).

[5] M. Christandl et al., Phys. Rev. Lett. 93, quant$\mathrm{ph} / 0411020$

[6] T. Shi, Y. Li, Z. Song, C.P. Sun, Phys. Rev. A 71, 032309
(2005).

[7] G. De Chiara, D. Rossini, S. Montangero, R. Fazio, quant-ph/0502148.

[8] A. Kay, M. Ericsson, quant-ph/0504063.

[9] Y. Li et al., Phys. Rev. A 71, 022301 (2005).

[10] M.B. Plenio, F.L. Semiao, New J. Phys. 7, 73 (2005).

[11] F. Verstraete, M.A. Martin-Delgado, J.I. Cirac, Phys. Rev. Lett. 92, 087201 (2004).

[12] T.J. Osborne, N. Linden, Phys. Rev. A 69, 052315 
(2004).

[13] D. Burgarth, S. Bose, quant-ph/0406112

[14] D. Burgarth, V. Giovannetti, S. Bose, quant-ph/0410175

[15] D. Burgarth, S. Bose, quant-ph/0502186

[16] V. Subrahmanyam, Phys. Rev. A 69, 034304 (2004).

[17] J. Zhang, quant-ph/0503199

[18] A. Romito, R. Fazio, C. Bruder, quant-ph/0408057.

[19] V. Giovannetti, R. Fazio, Phys. Rev. A 71, 032314
(2005).

[20] H.L. Haselgrove, quant-ph/0404152

[21] Y. Li, Z. Song, C.P. Sun, quant-ph/0504175.

[22] M. Paternostro, G.M. Palma, M.S. Kim, G. Falci, Phys. Rev. A 71, 042311 (2005).

[23] L. Amico et al., Phys. Rev. A 69, 022304 (2004). 